\title{
The Koebner phenomenon in seborrheic pemphigus
}

\section{Siham Belmourida, Mehdi Khallayoune, Palamino Hind, Mariame Meziane, Nadia Ismaili, Laila Benzekri, Badredine Hassam, Karima Senouci}

\author{
Department of Dermatology-Venerology, Mohammed V University, Ibn Sina Hospital, Rabat, Morocco
}

Corresponding author: Dr. Siham Belmourida, E-mail: belmouridasiham2017@gmail.com

Sir,

The Koebner phenomenon (KP) manifests itself as lesions typical of a certain dermatosis in areas of healthy skin that were previously stimulated by various types of trauma $[1,2]$. Herein, we present the case of a patient with seborrheic pemphigus initially developed on a surgical scar.

A 25-year-old female, who underwent Cesarean section six months earlier for fetal distress, was hospitalized for a rash initially localized on the Cesarean section, which then gradually spread. A clinical examination revealed good general condition but erythematous squamous and crusty lesions on the surgical scar (Fig. la), trunk (Fig. 1b), and back. Oily dander was also noted with erythema on the scalp (Fig. lc). The mucous membranes and nails were without abnormality, and the Nikolsky sign was absent.

A skin biopsy (Fig. 2) and direct immunofluorescence were in favor of superficial pemphigus. Anti-intercellular substance antibodies were at 1280. Both anti-nuclear antibodies and native anti-DNA antibodies were negative. In light of these results, the diagnosis of seborrheic pemphigus was reached and prednisone treatment was initiated at a rate of $1.5 \mathrm{mg} / \mathrm{kg} /$ day with good progress.

The Koebner phenomenon (KP) is one of the most wellknown entities in dermatology, first described in 1876 by Heinrich Koebner in only psoriatic patients. This isomorphous phenomenon is now well known in psoriasis, vitiligo, lichen planus, and Darier's disease [1,2]. Some rare cases have been described in pemphigus vulgaris but, to the best of our knowledge, our patient is the first case of the KP in seborrheic pemphigus.

The pathogenesis of the KP is still poorly understood but may involve cytokines, stress proteins, adhesion molecules, and auto-antigens [2]. In our patient, the KP might be explained by the fact that scar tissue tends to develop koebnerization more easily, which in turn is explained by changes in vascularization and chronic mast cell infiltrate that affects the regional endothelium.
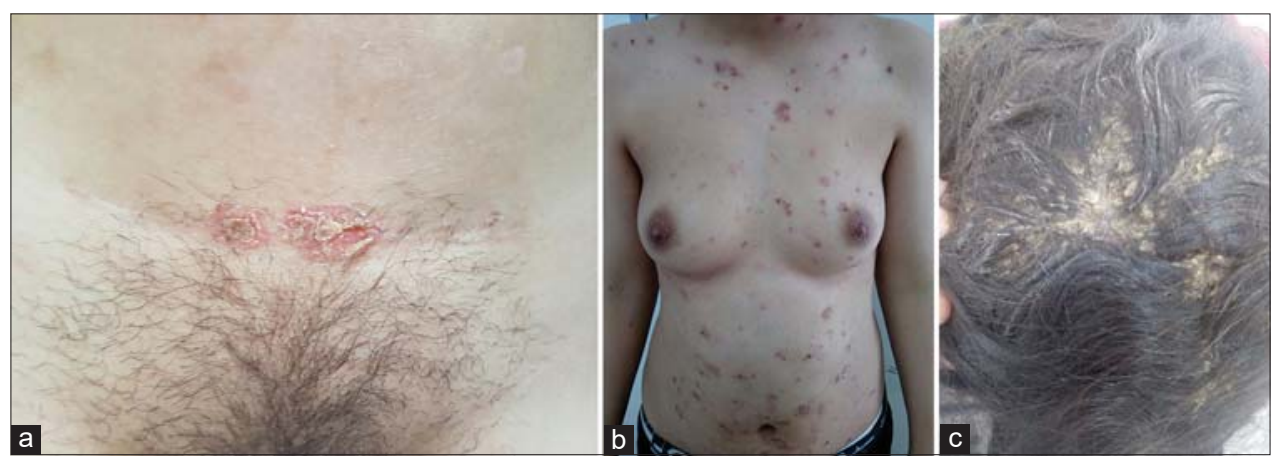

Figure 1: (a) Superficial pemphigus lesions on the surgical scar, illustrating the Koebner phenomenon, (b) Superficial pemphigus lesions on the trunk (c) Oily dander with erythema on the scalp.

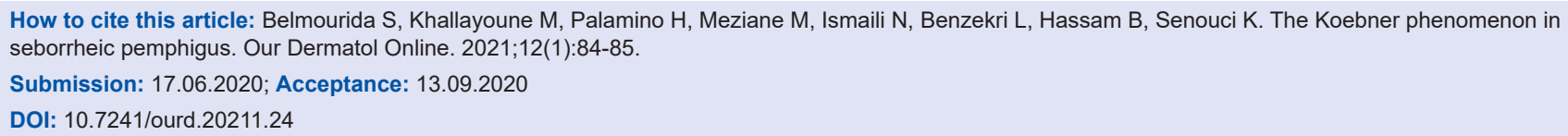




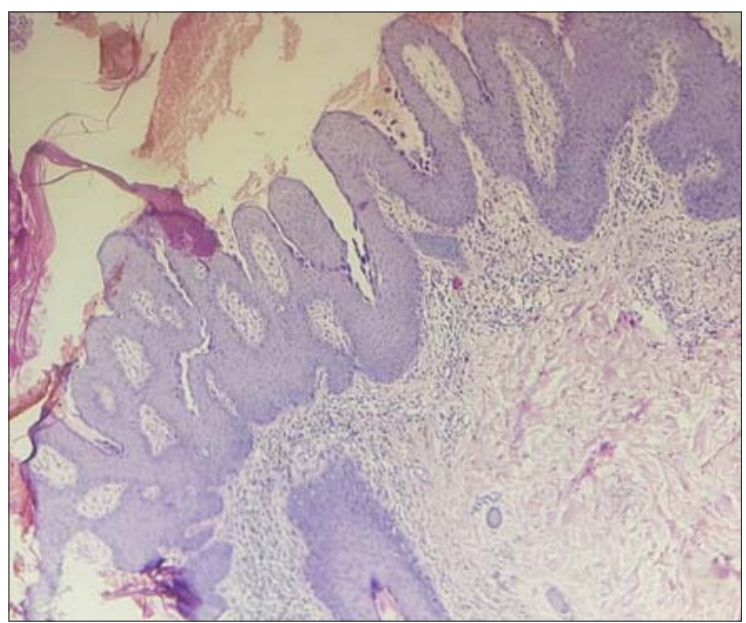

Figure 2: Histological image showing superficial acantholysis and a papillomatous epidermis.

Unlike pemphigus vulgaris, seborrheic pemphigus is clinically characterized by erythematous squamous lesions rather than bullous lesions. Thus, lesions of seborrheic pemphigus may more closely mimic postoperative wound infections and contact eczema. This can lead to delayed diagnosis, especially in patients with no history of pemphigus [3].

Therefore, exercising clinical suspicion of the various dermatoses with koebnerization that can follow skin surgery may allow for a more rapid diagnosis and immediate management.

\section{Consent}

The examination of the patient was conducted according to the principles of the Declaration of Helsinki.

The authors certify that they have obtained all appropriate patient consent forms, in which the patients have given consent for images and other clinical information to be included in the journal. The patients understand that their names and initials will not be published and due effort will be made to conceal their identity, but that anonymity cannot be guaranteed.

\section{REFERENCES}

1. Al Hamzawi NK. Lichen planus arising through the Koebner phenomenon in areas of Hijama. Our Dermatol Online. 2019;10:198-9.

2. Yamamoto T. Köbner phenomenon in systemic lupus erythematosus. Our Dermatol Online. 2019;10:101-2.

3. Daneshpazhooh M, Fatehnejad M, Rahbar Z, Balighi K, Ghandi N, Ghiasi M, et al. Trauma-induced pemphigus: a case series of 36 patients. J Dtsch Dermatol Ges. 2016;14:166-71.

Siham Belmourida, et al. This is an open access article distributed under the terms of the Creative Commons Attribution License, which permits unrestricted use, distribution, and reproduction in any medium, provided the original author and source are credited.

Source of Support: Nil, Conflict of Interest: None declared. 